\title{
Reinvestigation of the structure of low cristobalite
}

\author{
By W. A. Dollase
}

Massachusetts Institute of Technology, Cambridge, Massachusetts

With 3 figures

(Received November 16, 1964)

\begin{abstract}
Auszug
Verzwillingte Kristalle von $\alpha$-Cristobalit von Ellora, Hyderabad, Indien haben die Raumgruppe $P 4_{1} 2_{1}$ (oder $P 4_{3} 2_{1}$ ) und die Gitterkonstanten $a=4,97_{8} \AA$ und $c=6,94_{8} \AA$. Eine dreidimensionale Verfeinerung mittels Ausgleichsrechnung bestätigte auf Grund von 110 integrierten Intensitäten (unter Berücksichtigung der Verzwillingung die von NIEUwENkamP bestimmte Struktur; der $R$-Wert wurde auf $4,4 \%$ verbessert. Die Parameter sind $u=0,30004 \pm 0,00033$ für $\mathrm{Si}$ und $x=0,23976 \pm 0,00089, y=0,10324 \pm 0,00085$ und $z=0,17844$ $\pm 0,00052$ für $\mathrm{O}$. Daraus folgen die Si-O-Abstände $1,601 \pm 0,004$ und 1,608 $\pm 0,004 \AA$.

Abstract

Twinned crystals of low cristobalite from Ellora, Hyderabad, India were found to have space group $P 4_{1} 2_{1}$ (or $P 4_{3} 2_{1}$ ) and cell dimensions $a=4.97_{8}$ and $c=6.94_{8} \AA$. A three-dimensional full-matrix least-squares refinement based upon 110 integrated intensities which were corrected for the effects of twinning, confirmed NIEUWENKaMP's original structure and reduced the final $R$ value to $4.4 \%$. The refined set of four positional parameters are; $u=.30004 \pm .00033$, $x=.23976 \pm .00089, y=.10324 \pm .00085, z=.17844 \pm .00052$. These refined parameters lead to $\mathrm{Si}-\mathrm{O}$ distances of $1.601 \pm .004$ and $1.608 \pm .004 \AA$.
\end{abstract}

\section{Introduction}

High cristobalite is the polymorph of $\mathrm{SiO}_{2}$ whose stability range occurs between $1470^{\circ}$ and the melting point of $1728^{\circ}$. Upon cooling, it tends to exist metastably down to a variable inversion temperature of about $268^{\circ}$ where it inerts to low cristobalite. The exact structures of the cristobalites are necessary to the understanding of the silica system, silica polymorphism, and the large field of silicates having related structures. Accordingly, the cristobalite structures have received considerably attention. Besides the early direct crystal-structure 
determinations, which are reviewed below, there have been a number of later, less direct studies which have yielded structural implications. The results of this latter group have been included, in the main, in the recent compilation of FroNDEL (1962).

\section{Previous structural studies}

The first structural study of high cristobalite was made by R. W. G. Wyckofr (1925) using powder photographs taken at $290^{\circ}$ and $430^{\circ}$. The proposed structure belonged to the isometric space group $F d 3 m$. The agreement between the 16 observed and calculated intensities, some of which were the sum of superposed powder lines, was fair, and could be improved somewhat by increasing the scattering power of the silicon atom relative to that of the oxygen atom. This structure, as well as all following cristobalite-structure proposals, showed an infinite three-dimensional network of more or less regular $\mathrm{SiO}_{4}$ tetrahedra with all corners shared. The silicon-oxygen distance in WyckofF's structure was $1.54 \AA$ and the $\mathrm{Si}-\mathrm{O}-\mathrm{Si}$ angle between joined tetrahedra was $180^{\circ}$.

'TOм F. W. ВАRTH (1932a) suggested a high-cristobalite structure belonging to space group $P 2_{1} 3$ on the basis of some extra lines in the powder photographs (taken at temperatures up to $500^{\circ}$ ) which are not allowed by WyckofF's space group. The agreement between the $17 \mathrm{ob}$ served and calculated intensities was rather poor. Two-thirds of the intensities actually observed were the sums of two or more nonequivalent reflections. The $\mathrm{Si}-\mathrm{O}$ distances ranged from 1.58 to $1.69 \AA$.

Later in the same year BARTH (1932b) made the first structural study of low cristobalite. Reasoning that it must belong to a subgroup of $P 2_{1} 3$, and ruling out, on optical grounds, systems more symmetrical than orthorhombic, he arrived at space group $P 2_{1} 2_{1} 2_{1}$. With this space group and with the pseudoisometric cell dimensions, nearly all the lines of the powder photographs he used were the sums of two or three nonequivalent reflections. The agreement between the $13 \mathrm{ob}-$ served and calculated intensities was fair. The $\mathrm{Si}-\mathrm{O}$ distances for this model ranged from 1.50 to $1.75 \AA$.

W. Niedwenkayp (1935) reinvestigated low cristobalite and suggested a structure belonging to the tetragonal space group $P 4_{1} 2_{1}$. The structure determination was made by qualitative estimation of intensities from rotation photographs made with twinned material. Due to the fortuitous cell dimensions many of the reflections were superposed on the photographs making it possible to estimate only 
the combined intensities of these pairs. The two $\mathrm{Si}-\mathrm{O}$ distances of this model were both $1.59 \AA$, and the $\mathrm{Si}-\mathrm{O}-\mathrm{Si}$ angle was $146^{\circ}$.

NieUWENKaMP (1937) also reinvestigated high cristobalite and proposed a third structure. From rotation photographs taken at $270^{\circ}$, using the same crystals as in the low-cristobalite study, he determined the space group to be $F d 3 m$, the same as that proposed by WrckofF. The best fit of the 25 observed and calculated intensities was with the oxygen atom disordered around the circumference of a circle of radius $0.4 \AA$. This circle was oriented normal to the $\mathrm{Si}-\mathrm{Si}$ axis of joined tetrahedra. Nieuwenkamp's model gave fair agreement of intensities and could account for WYcKofF's observation of the low scattering power of the oxygen atom. The $\mathrm{Si}-\mathrm{O}$ distance in this model was $1.59 \mathrm{~A}$ and the $\mathrm{Si}-\mathrm{O}-\mathrm{Si}$ angle was $151^{\circ}$.

On the basis of these several structure determinations, which are characterized by meager, ambiguous data leading to conflicting results, the structures of the cristobalites are, at best, somewhat uncertain and merit reinvestigation. It is the purpose of this paper to report the results of the reinvestigation of the low-cristobalite structure.

\section{Unit cell and space group}

Crystals of low cristobalite from Ellora, Hyderabad, India were kindly made available from the Harvard Museum collection by Professor Clifford Frondel. A description of the material from this locality is given by Frondel (1962, page 282), and by van ValkenBURG and BUIE (1945).

The cell dimensions and systematic absences were determined from precession photographs, taking into account the twinning which is discussed below. The diffraction symbol is $4 / m m m P 4_{1} /-2_{1}-$, which determines the space group as $P 4_{1} 2_{1}$ or its enantiomorph, $P 4_{3} 2_{1}$. The

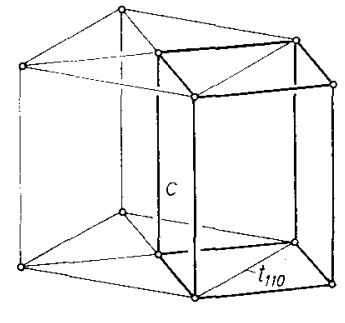

a)

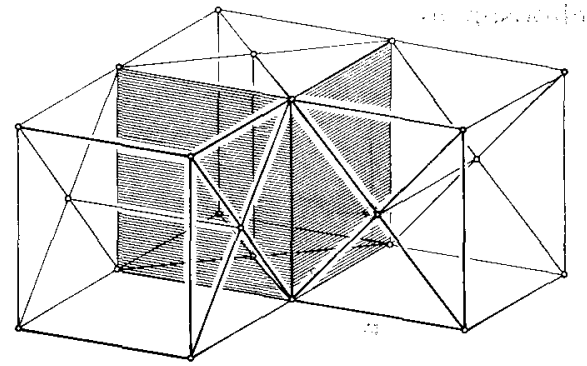

b)

Fig. 1 
cell dimensions, $a=4.97_{8}$ and $c=6.94_{8} \AA$, compare well with the values given by Frondel (1962, page 276).

When dealing with a primitive tetragonal lattice it is possible, though unconventional, to choose a $C$-centered cell. The relationship between the two cells is shown in Fig.1a. For the particular case of low cristobalite this $C$-centered cell is pseudoisometric. The discrepancy between the $c$-axis translation and $t_{110}(=a \sqrt{2})$ of the primitive cell, is only $1.3 \%$. This pseudoisometric cell is dimensionally equivalent to the high-cristobalite cell.

\section{Deseription of the twinning}

$\mathrm{X}$-ray examination of the few available crystals from this locality showed that each of them was twinned. The pattern of reflections given by $c$-axis precession photographs is illustrated in Fig. $2 a$. Besides the reflections attributable to the $P 4_{1} 2_{1}$ cell deseribed by NIEUWENKaMr (large circles), there were observed additional weak reflections (small circles) falling between the Nieuwenkamp-cell reflections. A possible supercell origin for these additional reflections can be discarded for the following reason. Their very low intensity would require a structure only slightly distorted from $P 4_{1} 2_{1}$ symmetry, whereas the new diffraction symbol (obtained when the additional reflections are treated as superstructure reflections) would require drastic deviation from this symmetry.

Although not observable on the precession photographs, further examination of the crystals on a counter diffractometer showed additional weak reflections satellitic to some of the Nieuwenkamp-cell reflections, as shown exaggerated in Fig. $2 b$. The location and number of the satellitic reflections as well as the extra reflections mentioned above can be accounted for by twinning. The twin operations may be choosen as $90^{\circ}$ rotations about the [110] and [110] directions of the

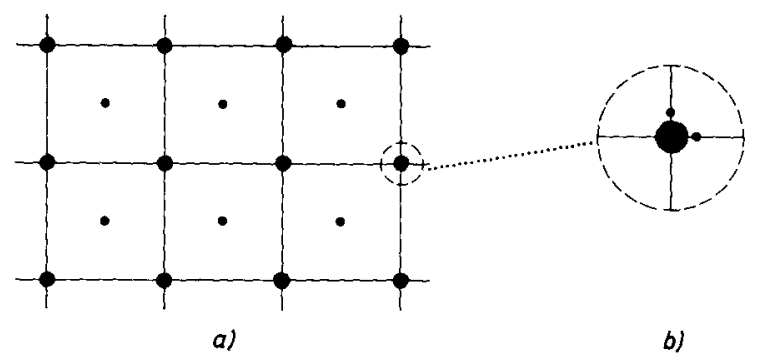

Fig. 2 
primitive tetragonal cell (see Fig. $1 a$ ). These two operations relate a reference twin individual with two other twin individuals, the three comprising the composite twin as shown in Fig. $1 b$.

On the basis of the relationship between the twin individuals the indices of the additional reflections may be tentatively assigned. The intensity of a reflection from one individual may then be plotted against the intensity of the same reflection from a second individual
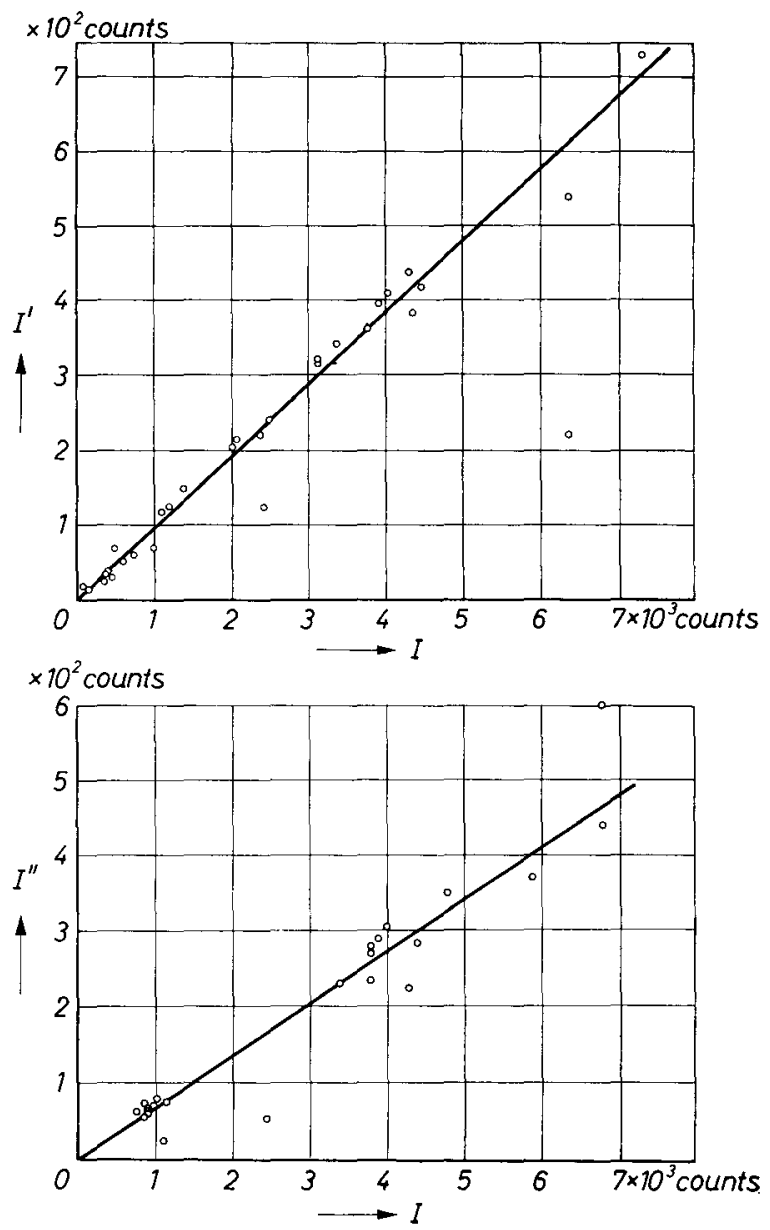

Fig. 3. Graphs of the integrated intensity of nonsuperposed reflections from one twin individual plotted against the integrated intensity of the same nonsuperposed reflections from a second twin individual. $I, I^{\prime}$ and $I^{\prime \prime}$ represent the integrated intensity of reflections from the first, second, and third twin individuals, respectively. 
for all the single, nonsuperposed reflections which are individually measureable. The linearity of such a plot confirms the indexing of the additional reflections and thus confirms the relationship between the twin individuals. Figure 3 shows such plots made with the crystal used for intensity measurements. The precision of measurement of the additional reflections is low due to their weak intensities. Taking this into consideration, the plots are satisfactorily linear.

\section{Collection of intensity data}

A small pseudooctahedron, $0.15 \mathrm{~mm}$ on a side, was selected for intensity measurements. An equi-inclination diffractometer employing a proportional counter was used to collect integrated intensities with nickel-filtered $\mathrm{Cu} K$ radiation. The $\lambda / 2$ harmonic was removed by pulseheight analysis.

The single, nonsuperposed reflections presented no problem in their measurement. On the other hand, the composite, superposed reflections are somewhat spread out as can be seen from Fig. $2 b$. To ensure collection of the entire composite intensity an extended $\omega$ scan, as well as a large counter aperture, was used.

About 200 reflections, including a number of the same reflections from the different twin individuals, were collected. The data were corrected in the usual way. Since $\mu r=0.6$, no absorption correction was applied.

\section{Correction of the data for twinning}

It is possible to extract a set of individual intensities from the composite reflections if the volumes of the twin individuals are not all equal. In order to obtain a sufficiently large and random set of intensities for least-squares refinement it is desireable to use this extracted set of intensities as well as the intensities of the nonsuperposed reflections. Since the lattices of the twin individuals in the twinned position mismatch appreciably, it may be assumed that there is negligible coherence of the diffracted beam and that the intensity of the composite reflections is the sum of the intensities of the contributing individual reflections. Taking into consideration the volumes of the twin individuals, this relation between the composite and individual reflections is

$$
C_{h k l}=t I_{h k l}+u I_{k^{\prime} k^{\prime} l^{\prime}}+v I_{h^{\prime \prime} k^{\prime \prime} l^{\prime \prime}}
$$

where $t, u$ and $v$ are the volumes of twin individuals. Using the orientation of the largest individual of the twin as the reference orientation, 
the indices of the individual reflections are found by operating on the indices of the composite reflection with the matrices

$$
\left(\begin{array}{lll}
1 & 0 & 0 \\
0 & 1 & 0 \\
0 & 0 & 1
\end{array}\right) \quad\left(\begin{array}{lll}
\frac{1}{2} & \frac{1}{2} & \frac{1}{2} \\
\frac{1}{2} & \frac{1}{2} & \frac{1}{2} \\
1 & \frac{1}{2} & 0
\end{array}\right) \quad\left(\begin{array}{cccc}
\frac{1}{2} & \frac{1}{2} & \frac{1}{2} \\
\frac{1}{2} & \frac{1}{2} & \frac{1}{2} \\
\frac{2}{1} & \frac{1}{2} & 0
\end{array}\right) .
$$

These are respectively the unit matrix and the matrices representing the twin operations given above. In practice, the volumes $t, u$ and $v$ are difficult to determine directly, so the volume ratios, $1=t / t, \mu=u / t$ and $\nu=v / t$, are used. These may be accurately estimated from the intensity ratios of the nonsuperposed reflections plotted in Fig. 3. The upper plot gives $\mu=0.094$ and the lower plot gives $v=0.064$.

Although the individual intensities may be obtained by the normal methods of solving a set of simultaneous equations, for computer solution an iterative method is advantageous and was employed. The recursive formula used was

$$
{ }^{i+1} I_{h k l}=C_{h k l}-\mu^{i} I^{\prime}{ }_{h^{\prime} k^{\prime} l^{\prime}}-\nu^{i} I^{\prime \prime}{ }_{h^{\prime \prime} k^{\prime \prime} l^{\prime \prime}},
$$

where the left superscript refers to the $i$ th approximation of the term which bears the superscript. The starting approximation

$$
{ }^{1} I_{h k l}=C_{h k l}-\frac{\mu}{1+\mu+\nu} C_{h^{\prime} k^{\prime} l^{\prime}}-\frac{v}{1+\mu+\nu} C_{h^{\prime} k^{\prime \prime} l^{\prime \prime}}
$$

was employed. This method has the disadvantage that the convergence rate depends upon the ratios of the individual intensities, but in this case, due to the very small magnitude of $\mu$ and $\nu$, uniform convergence was reached in five cycles.

\section{Refinement}

After correcting for the effects of twinning, a total of 110 reflections with $\left|F_{0}\right|>0$ were available for least-squares refinement. The number of observations per variable is greater than 15 . The full-matrix leastsquares refinement program written by C.T. PREwITT was used. Scattering curves for both neutral and half-ionized atoms were tried without any significant change in the results. An equal-weighting scheme was employed. The original atom coordinates of NieuwerKAMP, together with the best fit of scale factor and isotropic temperature factors, yielded an $R$ value of $13 \%$. Six cycles of refinement varying all parameters led to convergence and a final $R$ value of $4.4 \%$. The final observed and calculated structure amplitudes are given in 
Table 1. During the refinement the silicon atom oscillated to both sides of the Nieuwenkamp value but finally settled within one standard deviation of his value.

The final atomic parameters are given in Table 2 along with those of Nieuwenkamp. The silicon atom occupies the position with site symmetry 2 in space group $P 4_{1} 2_{1}$ while the oxygen atom lies in the general position. The magnitudes of the temperature factors should not be regarded as very significant as the correlation factors between these values and the scale factor are 0.82 and 0.64 . The usual interaction of these dependent variables is further aggravated in this case by the small number of temperature factors and the rather narrow range of $(\sin \theta) / \lambda$ values. The correlation factor between the two temperature factors is smaller, implying that the ratio of the temperature factors is more meaningful than their individual magnitudes. The value of this ratio for low cristobalite is similar to those observed in other silicate refinements.

There are two independent silicon-oxygen distances and four independent oxygen-silicon-oxygen angles in the tetrahedron. The refined coordinates yield the values given in Table 3 . The silicon-oxygensilicon angle between joined tetrahedra is also included. The difference between the $\mathrm{Si}-\mathrm{O}$ distances is not statistically significant. The deviations of the angles from the ideal tetrahedral angle are as much as three standard deviations and may be significant. 
Table 2. Final atomic parameters*

\begin{tabular}{l|l|l|l|l|l|l|l}
\hline & $x$ & $\sigma_{x}$ & $y$ & $\sigma_{y}$ & $z$ & $\sigma_{z}$ & $B$ \\
\hline $\mathrm{Si}$ & .30004 & .00033 & - & - & - & - & $0.83 \AA^{2}$ \\
$\mathrm{Si}_{N}$ & .300 & .005 & - & - & - & - & - \\
$\mathrm{O}$ & .23976 & .00089 & .10324 & .00085 & .17844 & .00052 & 1.55 \\
$\mathrm{O}_{\mathrm{N}}$ & .245 & .005 & .100 & .010 & .175 & .005 & -
\end{tabular}

* The subscript, $N$, refers to Niedwenkamp's original values.

Table 3. Interatomic distances and angles

\begin{tabular}{ll|ll}
\hline $\mathrm{Si}-\mathrm{O}_{1}$ & $1.601 \pm .004 \AA$ & $\mathrm{O}_{1}-\mathrm{Si}-\mathrm{O}_{2}$ & $111.5 \pm .3^{\circ}$ \\
$\mathrm{Si}-\mathrm{O}_{2}$ & $1.608 \pm .004$ & $\mathrm{O}_{3}-\mathrm{Si}-\mathrm{O}_{4}$ & $109.2 \pm .3$ \\
& & $\mathrm{O}_{1}-\mathrm{Si}-\mathrm{O}_{3}$ & $108.2 \pm .3$ \\
& & $\mathrm{O}_{1}-\mathrm{Si}-\mathrm{O}_{4}$ & $109.9 \pm .3$ \\
& & $\mathrm{Si}-\mathrm{O}-\mathrm{Si}$ & $146.8 \pm .3$
\end{tabular}

Acknowledgements. The author is indebted to Professor M. J. BUERGER for having suggested this study and for his councel during the course of the investigation. The computations were carried out at the M. I. T. Computation Center. This work was supported by a grant from the National Science Foundation.

\section{References}

Tom F. W. Barth (1932a), The cristobalite structures: I. High cristobalite. Amer. Jour. Sci. 23, 350-356.

TOM F. W. BARTH (1932b), The cristobalite structures: II. Low cristobalite. Amer. Jour. Sci. 24, 97-110.

Clifford Frondel (1962), The system of mineralogy, 7th ed., vol. III: Silica minerals. John Wiley and Sons, New York, New York.

W. Nreuwenkamp (1935), Die Kristallstruktur des Tief-Cristobalits $\mathrm{SiO}_{2}$. Z. Kristallogr. 92, 82--88.

- (1937), Über die Struktur von Hoch-Cristobalit. Z. Kristallogr. 96, 454-458.

A. VAN VALkEnbURG JR. and B. F. BUie (1945), Octahedral cristobalite with quartz paramorphs from Ellora Caves, Hyderabad State, India. Amer. Min. $30,526-535$.

R. W. G. WYCKoFy (1925), The crystal structure of the high temperature form of cristobalite $\left(\mathrm{SiO}_{2}\right)$. Amer. Jour. Sci. 9, 448-459. 